\title{
STUDY OF L-H TRANSITION AND PEDESTAL WIDTH BASED ON TWO-FIELD BIFURCATION AND FIXED POINT CONCEPTS
}

\author{
Boonnyarit Chatthong*, Thawatchai Onjun
}

\author{
School of Manufacturing Systems and Mechanical Engineering, Sirindhorn International Institute of \\ Technology, Thammasat University, Pathum Thani, Thailand \\ * corresponding author: boonyarit.chatthong@gmail.com
}

\begin{abstract}
The $\mathrm{L}-\mathrm{H}$ transition in magnetic confinement plasmas is investigated on the basis of concepts of two-field bifurcation and fixed-point stability. A set of heat and particle transport equations with both neoclassical and anomalous effects included is used to study ETB formation and also pedestal width and dynamics. It is found that plasmas can exhibit bifurcation where a sudden jump in the gradients can be achieved at the transition point corresponding to the critical flux. Furthermore, it is found that the transport barrier expands inward, whereby the radial growth of the pedestal initially appears to be superdiffusive but later slows down and stops. In addition, the time of barrier expansion is found to be much longer than the time that plasma takes to evolve from L-mode to H-mode. A sensitivity study is also performed, in which the barrier width is found to be sensitive to various parameters, e.g. heating, transport coefficients and suppression strength.
\end{abstract}

KEYWORDS: plasma; tokamak; fusion; L-H transition; bifurcation.

\section{INTRODUCTION}

Experimental observations in various magnetic confinement fusion devices have revealed that the formation of an edge transport barrier (ETB) results in a sudden transition from low-confinement mode (L-mode) to high-confinement mode (H-mode) with great improvement in plasma performance [1]. This improvement is necessary for future nuclear fusion machines, such as ITER [2]. Understanding the physics of the $\mathrm{L}-\mathrm{H}$ transition [3] is still a key issue in fusion research. Experimentally, plasmas make the transition to H-mode when the injected heat exceeds a threshold marked by the formation of a transport barrier located near the edge of the plasma with a relatively high gradient profile (pressure/density) [4]. Consequently, the core profile rises, resulting in enhanced fusion performance. It is therefore crucial to be able to explain the physical mechanisms of transport barrier formation and dynamics.

Although the underlying physics of the $\mathrm{L}-\mathrm{H}$ transition is still unclear, many hypotheses have been put forward based on the concept of suppression of the turbulent transport by the flow shear or/and the magnetic shear [5. It is known that turbulent transport can be stabilized by flow shear because of the breaking of a convection cell 6. Experimental results support the idea that turbulent fluxes can be reduced or quenched by a sheared flow in the transport barrier region [5, 7]. Consequently, an ETB is formed and the plasma makes an abrupt transition from L-mode to H-mode.

Some earlier research based on bistable s-curve bifurcation models 8-15] provided insights into qualitative aspects, and also into $\mathrm{L}-\mathrm{H}$ transition physics. These works described the $\mathrm{L}-\mathrm{H}$ transition using an $s$-curve graph in nonlinear flux versus a gradient space with both stable and unstable branches. The bifurcation model was introduced to explain particle and energy confinement in tokamaks 9. Ref. [11 utilized a simple one-field bifurcation model to portray the spatiotemporal behavior of the plasma, and found the hysteresis loop. Malkov and Diamond later applied this concept to analyze coupled heat and particle transport equations simultaneously, and illustrated that when the hyper-diffusion effect is included, the transition follows Maxwell's rule [12. Recently, the model has included heat and momentum density transports for an analytical study of the impact of external torque on the formation of an internal transport barrier (ITB) [13. Different tasks can also be taken. For example, in [14, bifurcation theory was used to explain the transition and also the dithering $\mathrm{H}$-mode.

This work attempts to reveal the fundamentals of the $\mathrm{L}-\mathrm{H}$ transition via a simple model of bifurcation approach, which could describe an overall view of the intrinsic bi-stability of the plasma. The variation of the system state, indicated as the pressure gradient with respect to a control parameter such as the heat flux, exhibits an s-curve shape. Intrinsic behaviours of ETB, including its dynamics and width, are also investigated. The study is therefore based on the assumption of an ELM-free plasma, i.e. any gradientlimit instability is neglected in order to study the growth or the expansion of a transport barrier.

This paper is organized as follows: brief descriptions of bifurcation and fixed-point concepts are presented in Section 2 numerical results of the $\mathrm{L}-\mathrm{H}$ transition and pedestal analysis are illustrated in Section 3 and conclusions are presented in Section 4 


\section{BIFURCATION MODEL AND FIXED-POINT ANALYSIS}

This section introduces the bifurcation model, and presents a conceptual and visual discussion of the local stability of a plasma and the dynamics of the $\mathrm{L}-\mathrm{H}$ transition, as well as its locations in the bifurcation diagram. A simplified version of the heat and particle transport equations, in slab geometry, can be expressed, respectively, in the form:

$$
\begin{gathered}
\frac{3}{2} \frac{\partial p}{\partial t}-\frac{\partial}{\partial x}\left[\chi_{\text {neo }}+\frac{\chi_{\text {ano }}}{1+\alpha\left(\nu_{E}^{\prime}\right)^{2}}\right] \frac{\partial p}{\partial x}=H(x, t), \\
\frac{\partial n}{\partial t}-\frac{\partial}{\partial x}\left[D_{\text {neo }}+\frac{D_{\text {ano }}}{1+\alpha\left(\nu_{E}^{\prime}\right)^{2}}\right] \frac{\partial n}{\partial x}=S(x, t),
\end{gathered}
$$

where $p$ is plasma pressure, $n$ is plasma density, $\chi_{\text {neo }}$ and $D_{\text {neo }}$ represent the neoclassical transport coefficients, $\chi_{\text {ano }}$ and $D_{\text {ano }}$ represent the anomalous transport coefficients, $\nu_{E}^{\prime}$ is the flow shear suppression, $\alpha$ is a positive constant representing the strength of the suppression, and $\mathrm{H}$ and $S$ are the thermal and particle sources, localized at the center and at the edge of the plasma, respectively. The formation of an ETB is a result of anomalous transport reduction caused by the flow shear effect [16]. Hence, the main ingredient for stabilizing the anomalous transport is the flow shear, which accounts for the known reduction of the turbulent transport by a sheared radial electric field [ $[$ ]. It couples the two transport equations according to the force balance equation, as shown in [12]:

$$
\nu_{E}^{\prime}=c \frac{E_{r}^{\prime}}{B} \approx-\frac{c}{e B n^{2}} p^{\prime} n^{\prime}
$$

Note that the contributions of the curvature, the toroidal and poloidal rotation are neglected here. Equations (1) and (2) can be rewritten as the time variation of the pressure and density as:

$$
\begin{aligned}
& \frac{\partial p}{\partial t}=H-\frac{\partial}{\partial x}\left[\chi_{\text {neo }}+\frac{\chi_{\text {ano }}}{1+\alpha\left(\nu_{E}^{\prime}\right)^{2}}\right] g_{p}, \\
& \frac{\partial n}{\partial t}=S-\frac{\partial}{\partial x}\left[D_{\text {neo }}+\frac{D_{\text {ano }}}{1+\alpha\left(\nu_{E}^{\prime}\right)^{2}}\right] g_{n},
\end{aligned}
$$

where $g_{p} \equiv-p^{\prime}$ and $g_{n} \equiv n^{\prime}$. Integrations of these two equations with respect to $x$ yield the followings:

$$
\begin{aligned}
& \dot{w}=Q-\left[\chi_{\text {neo }}+\frac{\chi_{\text {ano }}}{1+\alpha\left(\nu_{E}^{\prime}\right)^{2}}\right] g_{p}, \\
& \dot{\eta}=\Gamma-\left[D_{\text {neo }}+\frac{D_{\text {ano }}}{1+\alpha\left(\nu_{E}^{\prime}\right)^{2}}\right] g_{n},
\end{aligned}
$$

where $Q=\int H \mathrm{~d} x$ and $\Gamma=\int S \mathrm{~d} x$ are the heat and particle fluxes given to the plasma. These two equations represent the time variation flow of energy and particle contents through a flux surface with $\dot{w}=\partial w / \partial t$ and $\dot{\eta}=\partial \eta / \partial t$ defined as follows:

$$
\begin{array}{r}
\int p \mathrm{~d} x=\sum_{i} p_{i} \Delta x_{i}=\sum_{i} \frac{F_{i}}{A_{i}} \Delta x_{i}=\sum_{i} \frac{W_{i}}{A_{i}} \\
=\sum_{i} w_{i} \equiv w,
\end{array}
$$

$$
\begin{array}{r}
\int n \mathrm{~d} x=\sum_{i} n_{i} \Delta x_{i}=\sum_{i} \frac{N_{i}}{V_{i}} \Delta x_{i}=\sum_{i} \frac{N_{i}}{A_{i}} \\
=\sum_{i} \eta_{i} \equiv \eta,
\end{array}
$$

where $F_{i}$ is the total force acting on a flux surface area $A_{i}$ due to the plasma pressure $p_{i}, W_{i}$ is the work done by the pressure, $w$ is total work done per surface area or the energy density of the plasma within the flux surface, and $\eta$ is the particle surface density of the plasma. These are first-order nonlinear differential equations of the thermal and particle transport equation. They are coupled through the shear term of (3). In the transient limit $\dot{w}=\dot{\eta} \cong 0$, a simple decoupling technique can be applied as $D_{\text {ano }} g_{n} \times(6)-\chi_{\text {ano }} g_{p} \times(7)$, resulting in:

$$
g_{n}=\frac{\Gamma \chi_{\mathrm{ano}} g_{p}}{Q D_{\mathrm{ano}}+D_{\mathrm{neo}} \chi_{\mathrm{ano}} g_{p}-\chi_{\mathrm{neo}} D_{\mathrm{ano}} g_{p}} .
$$

This can be substituted in (6) to decouple the two fields.

From this point on, the analysis is based on the heat transport equation for the $\mathrm{L}-\mathrm{H}$ transition. A similar discussion can be carried out for the particle transport equation, as [17] has shown that there exist both heating power and density thresholds for the L-H transition. Physically, 6 represents the change of the energy density with time. It depends on both the heat flux and the pressure gradient. This can be seen in Figure 1, where each panel illustrates a $\dot{w}$ versus $g_{p}$ diagram (or $\dot{\eta}$ versus $g_{n}$ for the particle field) based on various $Q$ values (or $\Gamma$ ). Note that all the constants are arbitrarily chosen in this work, so quantitative values have no physical meaning. With respect to time evolution, different behaviours can happen to a local plasma gradient point on this figure. Firstly, in the regions where $\dot{w}>0(\dot{\eta}>0)$, the pressure gradient (density gradient) increases with time (rightwards arrow) because the plasma energy (density) increases. Secondly, in the regions where $\dot{w}<0(\dot{\eta}<0)$, the pressure gradient (density gradient) decreases with time (leftwards arrow) because the plasma energy (density) decreases. Lastly, if the point lies at $\dot{w}=0(\dot{\eta}=0)$, implying that it is in equilibrium, the gradients do not change; such points are called fixed points.

These three behaviours of the local plasma gradients allow possibility to analyze the stability properties of the plasma. Panel (a) of Figure 1 shows the curve at relatively low fluxes value. Apparently, only one stable fixed point can be observed at a relatively low pressure gradient, because any deviation from it will bring it back, as shown by the arrows. Panel (b) shows the case where the fluxes reach their first critical values ( $Q_{1, \text { crit }}$ and $\left.\Gamma_{1, \text { crit }}\right)$, another half-stable fixed point appears. At higher fluxes, as shown in panel (c), the new fixed point splits into two fixed points, making total of three fixed points: two stable and one unstable. Panel (d) shows the case where the fluxes are equal to 

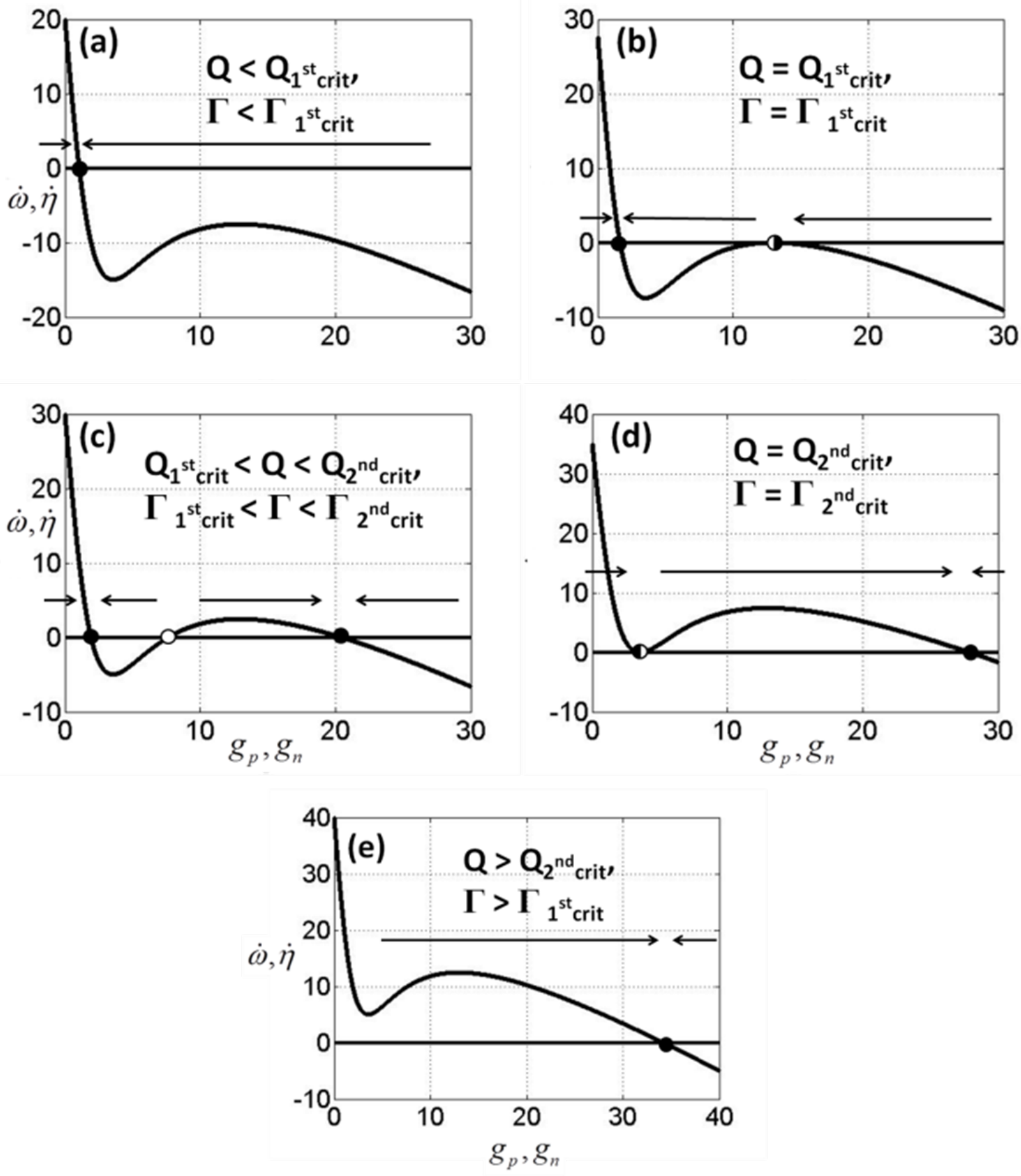

FiguRE 1. Fixed points for each heat/particle flux value and their stabilities: a solid dot for stable fixed points, an open dot for unstable fixed points, and a semi-open dot for semi-stable fixed points.

the second critical values $\left(Q_{2}\right.$,crit and $\Gamma_{2}$,crit $)$, the two fixed points on the left merge to a single half-stable point. Panel (e) shows the case where the second critical fluxes are exceeded, the half-stable point is destroyed, so only one stable point at relatively high gradients exists.

Dynamics of local gradients can be described using a graphical interpretation. The stability analysis method can lead to understanding of the $\mathrm{L}-\mathrm{H}$ forward transition and the $\mathrm{H}-\mathrm{L}$ back transition. In particular, the fluxes can be treated as independent variable, which can be changed. As they are increased or de- creased, the qualitative behaviour, e.g. the stability of fixed point, of the plasma system can be altered. The important assumption used throughout this paper is that the plasma relaxation time is sufficiently small. Figure 2 shows the fluxes versus gradients space of the fixed points and their stability for each fluxes value. Note that, a closed circle represents stable fixed points and an open circle represents unstable fixed points. They form traditional bifurcation diagram, similar to those shown in [11-13. Evidently, $Q_{L \rightarrow H}=Q_{2, \text { crit }}, \Gamma_{L \rightarrow H}=\Gamma_{2, \text { crit }}, Q_{H \rightarrow L}=Q_{1 \text {,crit }}$, and $\Gamma_{H \rightarrow L}=\Gamma_{1, \text { crit }}$. Essentially, the diagram shows 


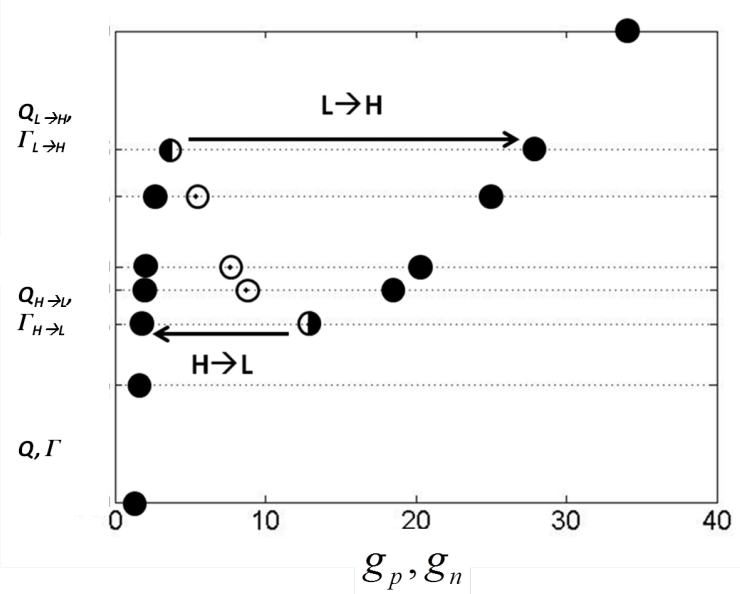

Figure 2. Bifurcation diagram illustrating two stable branches and one unstable branch with $\mathrm{L}-\mathrm{H}$ and $\mathrm{H}-\mathrm{L}$ transitions.

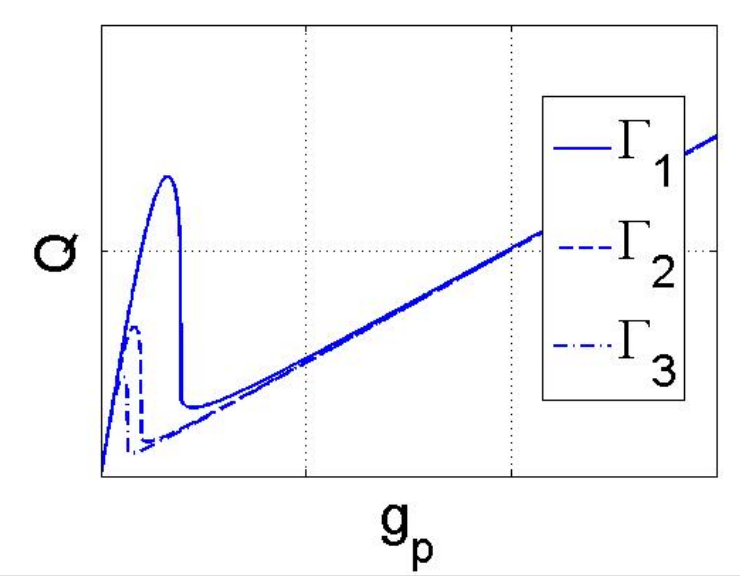

FiguRE 3. Bifurcation diagram of the pressure field at different particle flux values.

that the gradients depend non-monotonically on their respective fluxes. The two stable branches of the $s$-curve correspond to the low (L-branch) and high $(\mathrm{H}-$ branch) gradients. The third branch, containing only unstable fixed points, cannot be physically reached because the system is at unstable equilibrium. The onset of the $\mathrm{L}-\mathrm{H}$ transition is where the gradient jumps from a relatively low value to a high value if the flux is increased slightly above the $\mathrm{L}-\mathrm{H}$ threshold. The figure also shows that an $\mathrm{H}-\mathrm{L}$ back transition occurs when the flux is reduced below the $\mathrm{H}-\mathrm{L}$ threshold, with the gradient dropping from a relatively high value to a low value. The flux value of the $\mathrm{H}-\mathrm{L}$ transition is lower than that of the $\mathrm{L}-\mathrm{H}$ transition, implying hysteresis behaviour. Although each of the pressure and density fields can have a bifurcation diagram with threshold fluxes for the $\mathrm{L}-\mathrm{H}$ transition and the hysteresis loop, they interact with each other. This is illustrated in Figure 3, which shows three bifurcation curves on $Q$ versus $g_{p}$ space at different particle flux values $\left(\Gamma_{1}<\Gamma_{2}<\Gamma_{3}\right)$. As the particle flux is increased,
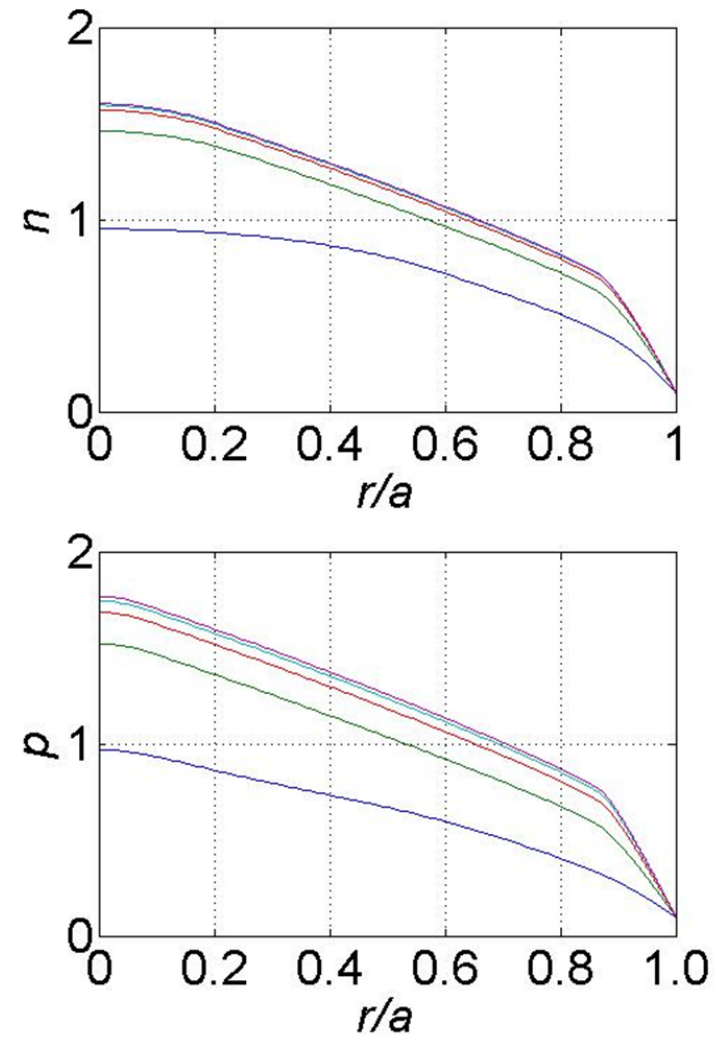

Figure 4. Plasma density (top) and pressure (bottom) profiles as a function of the normalized minor radius at times $200 \mathrm{~ms}$ apart.

the suppression strength due to the flow shear is also increased. It is therefore physically relevant that the requirement $Q_{L \rightarrow H}$ for the transition is less stringent.

\section{NumericAl RESUlts AND DISCUSSIONS}

In this section, the two transport equations (1) and (2) are solved simultaneously using a discretization method for the partial differential equation. Heat and particle sources are localized at the center and at the edge of the plasma, respectively, and they are assumed to be constant in time. The numerical results yield the time evolution of the plasma profiles i.e. the pressure, the density, and their gradients. The neoclassical transport coefficients are simply set to be constant, while the anomalous transport coefficients follow critical gradient transport model similar to that described in [18]:

$$
\begin{gathered}
\chi_{\mathrm{ano}}=c_{\chi}\left(p^{\prime}-p_{c}^{\prime}\right) \theta\left(p^{\prime}-p_{c}^{\prime}\right), \\
D_{\mathrm{ano}}=c_{D}\left(n^{\prime}-n_{c}^{\prime}\right) \theta\left(n^{\prime}-n_{c}^{\prime}\right),
\end{gathered}
$$

where $c_{\chi}$ and $c_{D}$ are constants, $p_{c}^{\prime}$ and $n_{c}^{\prime}$ are the critical gradients for pressure and density fields, respectively, and $\theta$ represents the Heaviside step function.

\subsection{Pedestal Dynamics}

This section illustrates the pedestal growth in the plasma. The crucial assumption to be noted here 

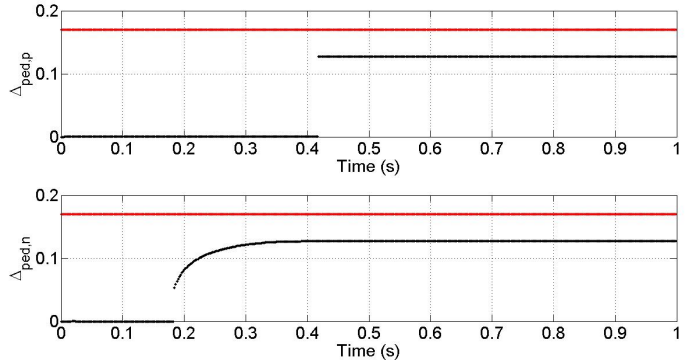

Figure 5. Pressure (top) and density (bottom) pedestal widths as a function of time for a constant source (horizontal line) scenario.

is that the pedestal is allowed to grow without any constraint, e.g. MHD instability, as the aim of this paper is to show the intrinsic property of the tokamak plasma system. Hence, these results are presumed to picture what would happen to the plasma and to its pedestal if the loss mechanism, e.g. ELM, can be controlled. Firstly, the two criteria (the minimum flux and the minimum diffusivities ratio) for the possibility of an $\mathrm{L}-\mathrm{H}$ transition according to bifurcation model are satisfied [19], so the plasma is ensured to reach the $\mathrm{H}$-mode in a steady state. Figure 4 demonstrates the time evolution profiles of plasma density and pressure at times approximately $200 \mathrm{~ms}$ apart. It shows that the plasma profiles make bigger increases early on. The change slows down as the plasma reaches a steady state. It can also be seen that the central pressure is almost doubled from L-mode to H-mode, whereas, the central density is increased by around $50 \%$. One other thing to note here is that the density profiles tend to be flatter in the plasma core. This makes sense, because the density flux is generated from the plasma edge, while the thermal flux comes from the plasma core.

It appears that when the gradient-limiting instability is neglected, the pedestal is intrinsically able to expand inward. This growth of the pedestal is shown in Figure 5, which illustrates the width of the pedestal as a function of time for both the pressure channel and the density channel. The heat and particle sources are assumed to be constant in time. Evidently, the pedestal is formed first on the density channel. The pedestal grows rapidly at first, and then it slows down and eventually reaches its steady state. It appears that the pedestal growth is strongly superdiffusive $\left(\Delta_{\text {ped }} \propto t^{b}, b>0.5\right)$, corresponding with a turbulent nature of the plasma, because in this phase the suppression effect is still low. Turbulent transport therefore plays a dominant role. Later, a wider region of the plasma is suppressed, so only neoclassical transport takes effect in the pedestal region, resulting in slower pedestal growth (subdiffusion or even lower). At some later time, a pedestal is also formed for the pressure. Two interesting points are worth mentioning here. Firstly, although the pedestals of
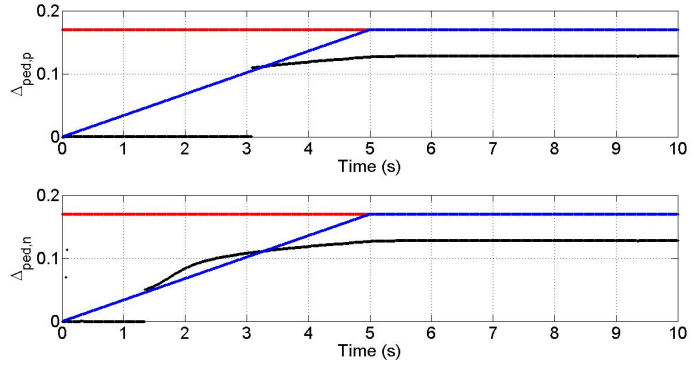

Figure 6. Pressure (top) and density (bottom) pedestal widths as a function of time for a heat ramping scenario.

the two channels do not form at the same time, they have the same width. This is likely to be explained by symmetry between the two transport equations. Secondly, the time it takes the plasma to evolve during $\mathrm{H}$-mode or the pedestal expansion time is around one order of magnitude slower than the time it takes for the plasma to evolve from the L-mode to the H-mode. This characteristic of the model is doubtful because, in the real tokamak plasma, instabilities at the edge cannot yet be controlled fully and efficiently. Moreover, in order to observe this behavior, it is necessary to make sure that the sole mechanism for plasma loss is via transport.

Figure 6 shows different scenarios when the heat source is no longer constant in time. Plasma heating (blue line) ramps up to a constant value, as in the previous scenario, while the particle source (red line) is kept constant at all times. Again, the pedestal is formed first in the density channel but the pedestal grows more slowly. It takes a longer time for the plasma to reach a steady state, because the heat is being ramped up. Eventually, the pedestal widths become the same as in the previous scenario in a steady state. This makes sense because in the end the heat and particle fluxes given to the plasma are the same.

\subsection{Pedestal Width}

This section focuses on an analysis of the pedestal width in a steady state. The relationships between the pedestal widths and various plasma parameters are shown in Figures 77 12. In these figures, the square bullets represent the pedestal width, which is the same for both pressure and density channels. The triangular bullets represent the central plasma pressure normalized to its value at the onset of the $\mathrm{L}-\mathrm{H}$ transition. The cross bullets represent the central plasma density normalized to its value at the onset of the $\mathrm{L}-\mathrm{H}$ transition.

Figure 7 shows the influence of the heat source on the plasma. It yields that there exists an $\mathrm{L}-\mathrm{H}$ transition threshold for heating. Below the threshold, there is no formation of a transport barrier. As the heating is increased above the threshold, the pedestal width 


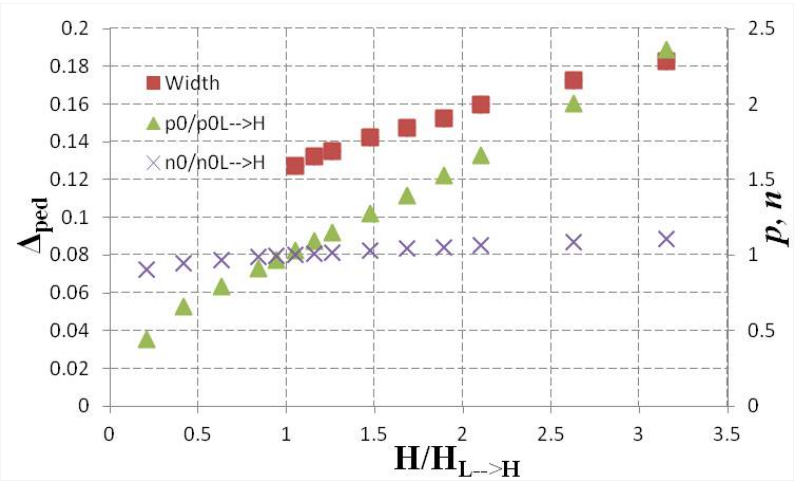

Figure 7. Pedestal width and central pressure and density in a steady state as a function of the heat source.

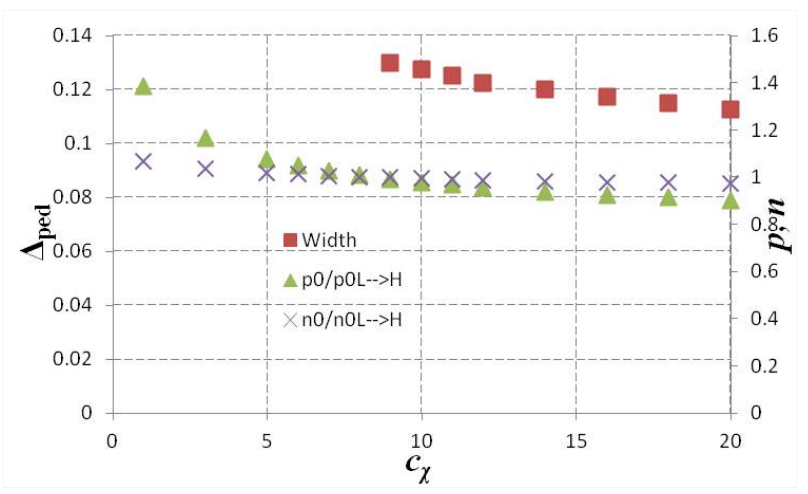

Figure 9. Pedestal width and central pressure and density in a steady state as a function of thermal anomalous transport.

widens but at a slower rate. Apparently, the change in the heat source has a greater effect on plasma pressure than on plasma density. Numerically for this particular case, the central pressure is increased to 3.76 times the lowest value, and the central density is increased to 1.18 times the lowest value, as the heat source is increased by 10 times the lowest value.

Figure 8 illustrates the influence of the particle source on the plasma. Similarly, this yields that there is also an $\mathrm{L}-\mathrm{H}$ transition threshold for particle flux. Below the threshold, there is no formation of a transport barrier. This finding and the heat source results are qualitatively in agreement with the stability analysis of Section 2 In contrast with the previous case, as the particle source is increased over the threshold, the pedestal width is in this case reduced. This can be explained by the suppression form of (3) used in the simulations. As the particle source is increased, the plasma density rises, resulting in a lower suppression value. Consequently, the plasma performance is reduced, while the pedestal width and also the pressure profile are decreased. Evidently, the change in particle source has a greater effect on plasma density than on plasma pressure. Numerically for this particular case, the central density is increased to 3.31 times the

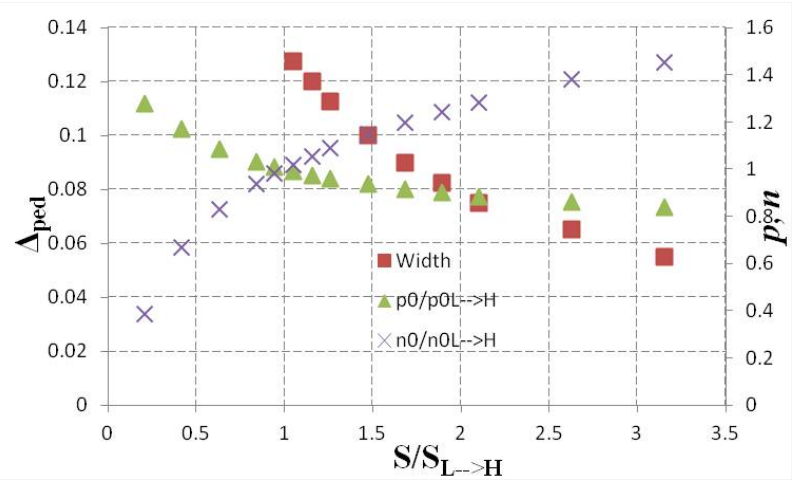

Figure 8. Pedestal width and central pressure and density in a steady state as a function of the particle source.

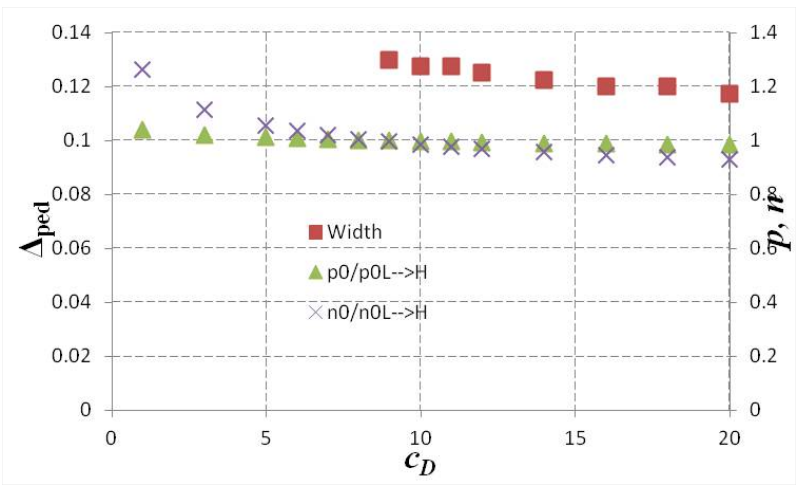

Figure 10. Pedestal width and central pressure and density in a steady state as a function of particle anomalous transport.

lowest value, and the central pressure is reduced to 0.69 times the lowest value, as the particle source is increased to 10 times the lowest value.

The effects of thermal anomalous transport are considered as shown in Figure 9. This study is carried out as a variation of the proportional constant $c_{\chi}$, from (11), which controls the strength of the thermal anomalous transport coefficient. Firstly, previous analysis in [12, 19] concluded that the $\mathrm{L}-\mathrm{H}$ transition is possible only if the ratio of anomalous transport over neoclassical transport exceeds a critical value. Generally, this value is in the order of 1 to 2. Physically, this condition always holds in a real plasma, because the anomalous transport is normally about 10 times higher in the ion channel and can even reach 100 times higher in the electron channel [20]. Figure 9 confirms the existence of this critical value for the realization of an $\mathrm{L}-\mathrm{H}$ transition. If the anomalous transport is too low, there is no formation of a transport barrier. Furthermore, as the strength of the anomalous transport is increased, the pedestal width narrows, and the central plasma pressure and the central plasma density are reduced. This makes sense: the plasma loss through transport is enhanced, so the plasma performance should be reduced. The 


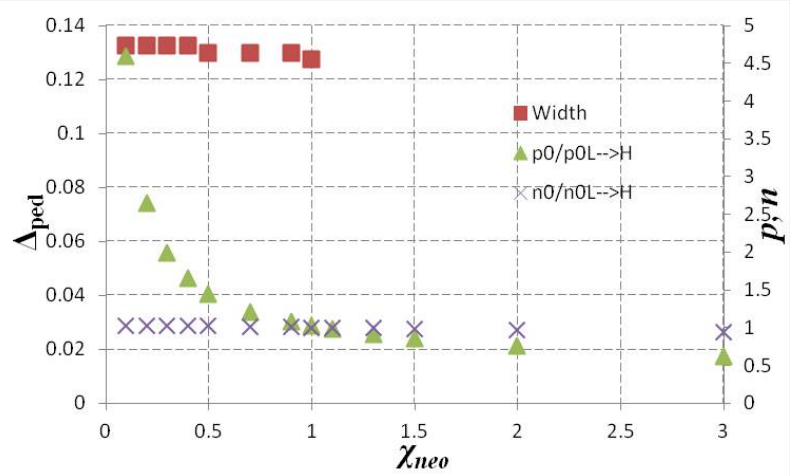

Figure 11. Pedestal width and central pressure and density in a steady state as a function of thermal neoclassical transport.

reductions of the profiles appear to be stronger in plasma pressure than in plasma density. Numerically for this particular case, the central pressure is reduced to 0.71 times the lowest value, and the central density is reduced to 0.93 times the lowest value, as the proportional constant $c_{\chi}$ is increased to 10 times the lowest value.

The effects of particle anomalous transport are shown in Figure 10 This study is carried out as a variation of the proportional constant $c_{D}$, from 12 , which controls the strength of the particle anomalous transport coefficient. Similarly, this figure also confirms the existence of a critical value for the possibility of an $\mathrm{L}-\mathrm{H}$ transition. If the anomalous transport is too low, there is no formation of a transport barrier. The results are similar to those presented in Figure 9 in which as the strength of the anomalous transport is increased, the pedestal width narrows, and the central plasma pressure and density are reduced. However, the reductions of the profiles appear to be stronger in plasma density than in plasma pressure. Numerically for this particular case, the central pressure is reduced to 0.96 times the lowest value, and the central density is reduced to 0.78 times the lowest value, as the proportional constant $\mathrm{cD}$ is increased to 10 times the lowest value.

The effects of thermal neoclassical transport are shown in Figure 11. where the transport coefficient is varied. The critical ratio for the possibility to obtain an $\mathrm{L}-\mathrm{H}$ transition is also shown here, because if the neoclassical transport is increased too greatly, the $\mathrm{H}$-mode cannot be reached. Also, as the strength of the thermal neoclassical transport is increased, the pedestal width narrows and the central plasma pressure and density are reduced. However, the reductions of the profiles appear to be stronger in plasma pressure. Numerically for this particular case, the central pressure is reduced to 0.23 times the lowest value, and the central density is reduced to 0.98 times the lowest value, as the thermal neoclassical transport coefficient is increased to 10 times the lowest value.

Figure 12 illustrates the effects of particle neoclassi-

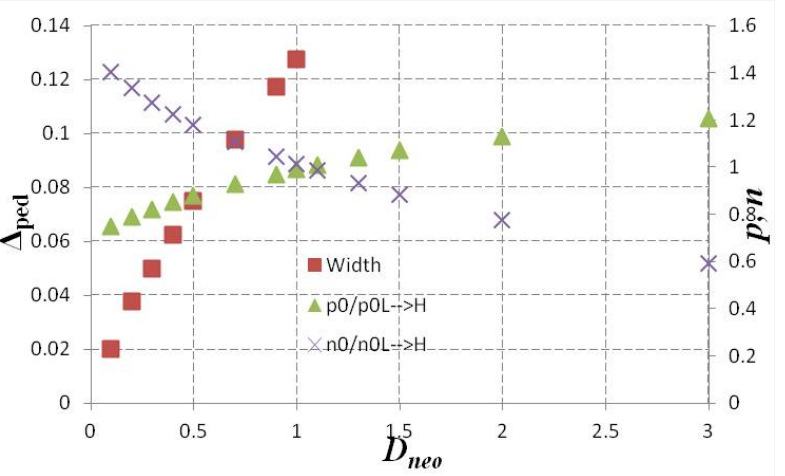

Figure 12. Pedestal width and central pressure and density in a steady state as a function of particle neoclassical transport.

cal transport on the pedestal width and on the central plasma values. The critical ratio for the possibility to obtain an $\mathrm{L}-\mathrm{H}$ transition is also evident here. Moreover, as the strength of the particle neoclassical transport is increased, the pedestal width is enlarged, the central pressure is increased and the central density is reduced. These results seem to be strange when compared with the results presented in Figure 11. The explanation is that when the strength of the particle neoclassical transport is increased, the plasma particle loss is enhanced. Subsequently, the plasma density is reduced, which increases the flow shear suppression, resulting in an increase in the pressure profiles and also in the pedestal width. The changes in the profiles appear to be stronger in plasma density. Numerically for this particular case, the central pressure is increased to 1.32 times the lowest value, but the central density is reduced to 0.72 times the lowest value, as the particle neoclassical transport coefficient is increased to 10 times the lowest value.

\section{Conclusions}

A numerical method has been used to simultaneously solve the two-field (heat and particle) transport equations. The transport effect considered here is a combination of the neoclassical transport, which is assumed to be constant, and the anomalous transport, which follows the critical gradient transport model. The suppression mechanism is the flow shear calculated from the shear of the radial electric field equation. An analytical study based on bifurcation and stability of fixed points shows that an abrupt increase in the local gradients occurs at the onset of an $\mathrm{L}-\mathrm{H}$ transition. This transition is also found to depend on the direction of heat ramping, where a backward $\mathrm{H}-\mathrm{L}$ transition can occur at lower fluxes than for a forward $\mathrm{L}-\mathrm{H}$ transition, implying hysteresis phenomena. Numerically, it is found that without gradient limiting instability, the pedestal width can initially expand superdiffusively and later subdiffusively. The time that the plasma takes for pedestal expansion is 
about one order of magnitude longer than it takes to transit from L-mode to H-mode. The pedestal tends to form first in the density channel, but in a steady state both pedestals have the same value. Furthermore, the pedestal width in a steady state and the central plasma pressure appear to be proportional to the heat source and the particle neoclassical transport, and inversely proportional to the particle source, the thermal and particle anomalous transports and the thermal neoclassical transport. The central plasma density appears to be proportional to the heat and particle sources, and inversely proportional to all plasma transports.

\section{ACKNOWLEDGEMENTS}

This work was supported by the Commission on Higher Education (CHE) and the Thailand Research Fund (TRF) under Contract No.RSA5580041. The authors greatly thank Y. Sarazin and IRFM, CEA. B Chatthong thanks the Royal Thai Scholarship and Thailand Institute of Nuclear Technology (TINT) for financial support and acknowledges the discussion at the 3rd APTWG, Korea 2013.

\section{REFERENCES}

[1] K. H. Burrell. Summary of experimental progress and suggestions for future work (H mode confinement). Plasma Phys. Control. Fusion 36:A291, 1994. DOI:10.1088/0741-3335/36/7a/043

[2] R. Aymar, P. Barabaschi, and Y. Shimomura. The ITER design. Plasma Phys. Control. Fusion 44:519, 2002. DOI:10.1088/0741-3335/44/5/304

[3] J. W. Connor, and H. R. Wilson. A review of theories of the L-H transition. Plasma Phys. Control. Fusion 42:R1, 2000. DOI:10.1088/0741-3335/42/1/201

[4] F. Wagner et al. Regime of Improved Confinement and High Beta in Neutral-Beam-Heated Divertor Discharges of the ASDEX Tokamak. Phys. Rev. Lett. 49:1408, 1982. DOI:10.1103/physrevlett.49.1408

[5] K. H. Burrell. Effects of E x B velocity shear and magnetic shear on turbulence and transport in magnetic confinement devices. Phys. Plasmas 4:1499-1518, 1997. DOI:10.1063/1.872367

[6] H. Biglari, P. H. Diamond, and P. W. Terry. Influence of sheared poloidal rotation on edge turbulence. Physics of Fluids B: Plasma Physics 2:1-4, 1990. DOI:10.1063/1.859529
[7] J.W. Connor et al. A review of internal transport barrier physics for steady-state operation of tokamaks. Nucl. Fusion 44:R1, 2004. DOI:10.1088/0029-5515/44/4/r01

[8] F. L. Hinton. Thermal confinement bifurcation and the L- to H-mode transition in tokamaks. Physics of Fluids B: Plasma Physics 3:696-704, 1991. DOI:10.1063/1.859866

[9] F. L. Hinton, and G. M. Staebler. Particle and energy confinement bifurcation in tokamaks. Physics of Fluids B: Plasma Physics 5:1281-1288, 1993. DOI:10.1063/1.860919

[10] G. M. Staebler, F. L. Hinton, and J. C. Wiley. Designing a VH-mode core/L-mode edge discharge. Plasma Phys. Control. Fusion 38:1461, 1996. DOI:10.1088/0741-3335/38/8/055

[11] V. B. Lebedev, and P. H. Diamond. Theory of the spatiotemporal dynamics of transport bifurcations. Phys. Plasmas 4:1087-1096, 1997. DOI:10.1063/1.872196

[12] M. A. Malkov, and P. H. Diamond. Analytic theory of $\mathrm{L}->\mathrm{H}$ transition, barrier structure, and hysteresis for a simple model of coupled particle and heat fluxes. Phys. Plasmas 15:122301, 2008. DOI:10.1063/1.3028305

[13] Hogun Jhang, S. S. Kim, and P. H. Diamond. Role of external torque in the formation of ion thermal internal transport barriers. Phys. Plasmas 19:042302, 2012. DOI:10.1063/1.3701560

[14] W. Weymiens et al. Bifurcation theory for the $\mathrm{L}-\mathrm{H}$ transition in magnetically confined fusion plasmas. Phys. Plasmas 19:072309, 2012. DOI:10.1063/1.4739227

[15] B. Chatthong, and T. Onjun. Investigation of toroidal flow effects on L-H transition in tokamak plasma based on bifurcation model. J. Phys.: Conf. Ser. 611:012003, 2015. DOI:10.1088/1742-6596/611/1/012003

[16] F. Wagner. A quarter-century of H-mode studies. Plasma Phys. Control. Fusion 49:B1, 2007. DOI:10.1088/0741-3335/49/12b/s01

[17] ASDEX Team. The H-Mode of ASDEX. Nucl. Fusion 29:1959, 1989. DOI:10.1088/0029-5515/29/11/010

[18] X. Garbet et al. Profile stiffness and global confinement. Plasma Phys. Control. Fusion 46:1351, 2004. DOI:10.1088/0741-3335/46/9/002

[19] B. Chatthong et al. Analytical and Numerical Modelling of Transport Barrier Formation Using Bifurcation Concept. 38th EPS Conference on Plasma Physics (Strasbourg, France), Paper P4.097. http://ocs.ciemat.es/EPS2011PAP/pdf/P4.097

[20] R C Wolf. Internal transport barriers in tokamak plasmas. Plasma Phys. Control. Fusion 45:R1, 2003. DOI:10.1088/0741-3335/45/1/201 